\title{
Finlande : de l'industrie lourde et sylvicole à une vibrante économie du savoir
}

Écrit par : Christophe André

Dernière mise à jour : 4 janvier 2019

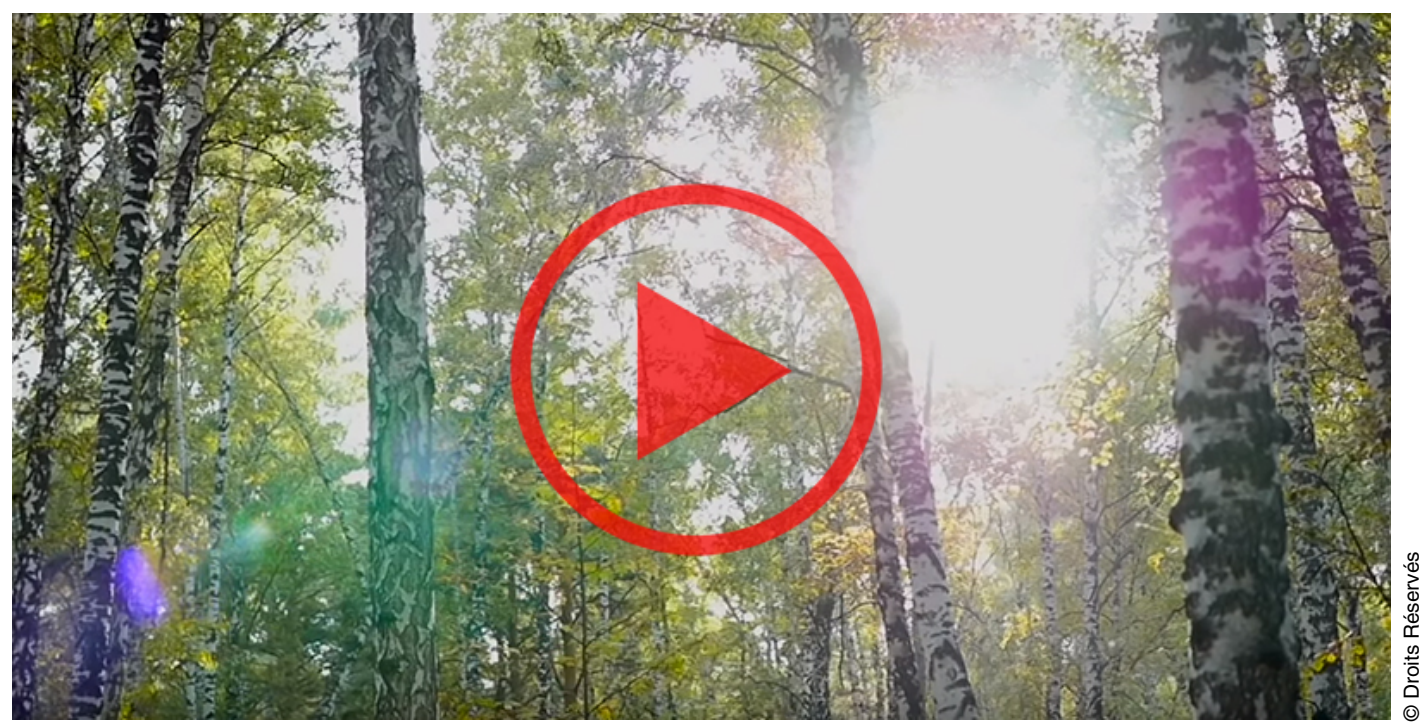

Video: Happy 50th anniversary, Finland at the OECD! https://oe.cd/obs/2zl

En un froid jour d'hiver 2014, j'attendais le Secrétaire général de l'OCDE, Ángel Gurría, et mes autres collègues, dans une limousine noire devant l'austère silhouette du parlement à Helsinki. Une ambiance digne des polars de John le Carré. Il se faisait tard. La délégation de l'OCDE que j'attendais rencontrait Alexander Stubb, alors ministre des Affaires européennes et du Commerce extérieur. M. Stubb, l'un des hommes d'État les plus europhiles et férus de technologie de son pays, allait peu après devenir Premier ministre. Que de chemin parcouru par la Finlande en cinquante ans !

Célébration du 50e anniversaire de l'adhésion de la Finlande à l'OCDE, le 28 janvier 2019 
En 1969, quand la Finlande rejoint l'OCDE, les tensions de la Guerre Froide battent encore leur plein. Le début de la décennie a été marqué par la crise des missiles de Cuba et la construction du mur de Berlin. La Finlande est un petit pays pris en étau entre les superpuissances soviétique et américaine. Le président Urho Kekkonen, qui restera à la tête du pays pendant un quart de siècle (1956-81), peine à maintenir un équilibre délicat entre l'Est et l'Ouest.

Pour la Finlande, la voie vers la prospérité et l'intégration dans l'Ouest est longue et semée d'embûches. La première Étude économique de l'OCDE sur la Finlande, publiée en 1969, remarque que « le poids important de l'agriculture, tant pour la production que pour l'emploi, demeure une caractéristique notable de l'économie finlandaise ». À l'époque, moins de $65 \%$ des Finlandais vivent en ville contre $80 \%$ de Suédois. L'agriculture emploie encore un quart de la main d'œuvre du pays. Plus d'un tiers des actifs travaillent dans le secteur secondaire, dominé par l'industrie du bois et la fabrication de pâtes et papiers, ainsi que par les industries métallurgiques (construction navale et d'appareils de levage), les transports, ou encore les équipements électriques. Le revenu par habitant en Finlande est $30 \%$ inférieur à celui de la Suède, où de nombreuses personnes émigrent dans l'espoir d'un travail mieux rémunéré.

Pendant les années 70 et 80 , le revenu par habitant de la Finlande rattrape rapidement ceux de la Suède et de l'Allemagne, l'écart se réduisant à moins de 10 \% (voir le graphique). Mais au début des années 90, la Finlande connaît une grave récession : son PIB chute de $10 \%$ entre 1990 et 1993. L'effondrement du bloc soviétique perturbe profondément les liens commerciaux. L'économie finlandaise est aussi mise à mal par une sévère crise bancaire, faute d'avoir mis en place un cadre de supervision suffisant après la déréglementation du marché des capitaux à la fin des années 80 . Au cours des années suivantes, la Finlande et d'autres pays nordiques renforcent leurs systèmes financiers. Hélas, nombreux sont les pays du monde qui, ne tirant pas les leçons de leur expérience, ont laissé les déséquilibres financiers s'accumuler jusqu'à provoquer la crise financière mondiale de 2008. 


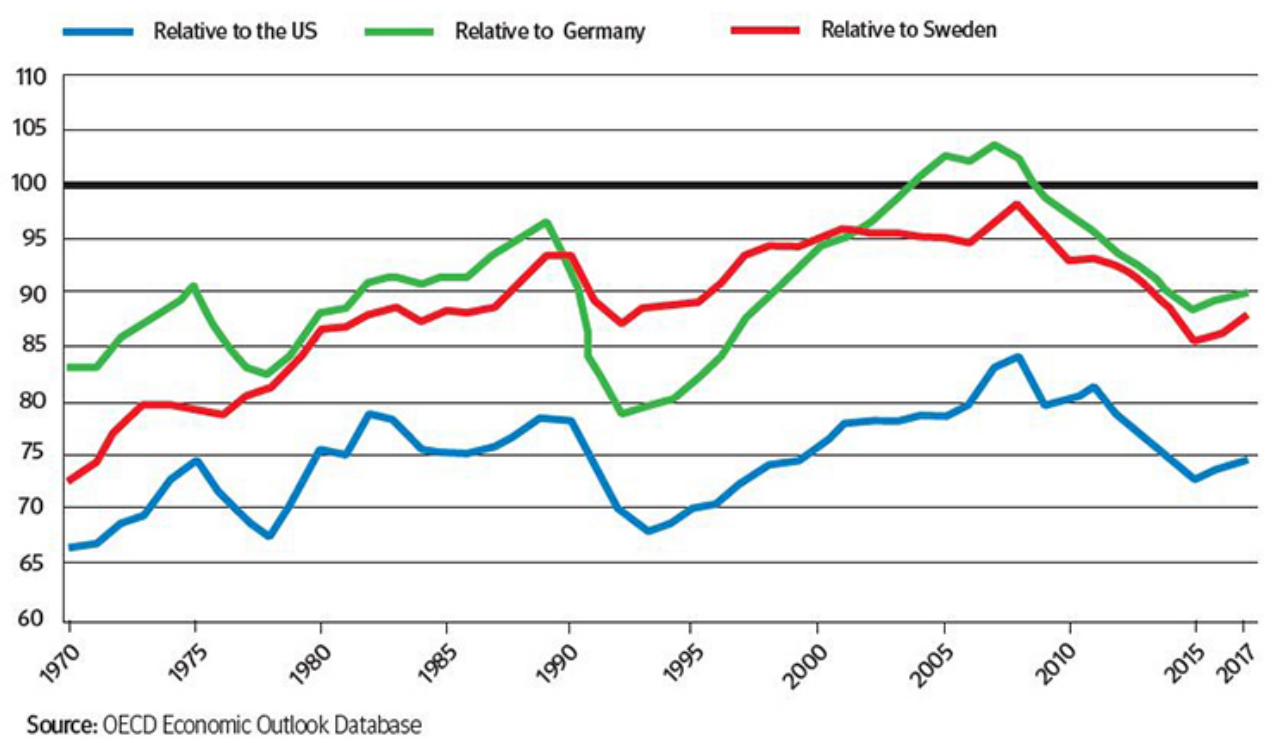

La crise des années 90 est douloureuse. Le taux de chômage frôle les 18 \% en 1994. Mais la Finlande fait montre d'une grande capacité de récupération, ou sisu, comme disent les Finlandais. L'économie reprend des couleurs et mue progressivement en une économie du savoir, comme l'illustre le succès spectaculaire de Nokia, devenu, dans les années 90, l'une des plus grandes marques de téléphones portables au monde. En 1995, la Finlande rejoint l'Union européenne, ce qui favorise les réformes économiques et donne de l'élan aux échanges. Quand la crise financière mondiale éclate, en 2008, le revenu par habitant de la Finlande a rattrapé celui de l'Allemagne et de la Suède. Mais, comme en 1990, la crise frappe durement le pays. Les effets du ralentissement économique mondial sont aggravés par l'effondrement de la branche mobile de Nokia, la forte baisse de la demande de papier et la récession qui frappe la Russie. Il faudra dix ans au PIB finlandais pour retrouver son niveau d'avant la crise. Mais la croissance économique et l'emploi ont retrouvé de l'allant ces trois dernières années, restaurant l'optimisme.

Quels bénéfices la Finlande a-t-elle tirés de son adhésion à l'OCDE ? Économistes et conseillers politiques soulignent l'importance politique de l'adhésion, cinquante ans plus tôt, à un club de démocraties occidentales, une décision qui a renforcé l'intégration internationale et la convergence. Ils saluent la contribution qu'apporte l'OCDE au débat public et politique finlandais dans un éventail de domaines toujours plus large en fournissant des données comparables, des références, des données scientifiques et des analyses comparatives. L'Organisation donne un point de vue externe précieux sur l'action publique, et encourage les échanges d'opinions entre analystes et responsables de l'élaboration des politiques des pays membres. En tant qu'économiste de l'OCDE, 
j'attache une grande valeur aux débats très ouverts, constructifs et stimulants avec mes homologues finlandais.

TCes derniers sont, à juste titre, fiers de leurs succès et de la contribution que leur pays apporte aux autres membres de l'OCDE. De l'éducation à l'innovation en passant par la croissance inclusive, les pratiques de la Finlande sont exemplaires dans bien des domaines. Elle affiche des scores parmi les meilleurs dans le cadre de l'enquête PISA, qui évalue les compétences et les connaissances des élèves de 15 ans dans le monde. Selon le tableau de bord européen de l'innovation, la Finlande est également chef de file en matière d'innovation. Les inégalités de revenu y sont parmi les plus faibles de l'OCDE, et l'égalité femmes-hommes parmi les plus fortes au monde : quand j'ai commencé à travailler à ma première Étude économique de l'OCDE sur la Finlande en 2011, Tarja Halonen était Présidente et Mari Kiviniemi, qui allait devenir Secrétaire générale adjointe de l'OCDE, était Première ministre. Mais, pour le premier pays à avoir élu des femmes au parlement, en 1907 (quand la Finlande était encore Grand-duché autonome de l'Empire russe), compter deux femmes aux postes les plus importants de l'État n'avait rien d'étonnant.

Aujourd'hui, la Finlande est confrontée à de nouveaux défis. L'un d'entre eux, et non des moindres, est de faire face au vieillissement rapide de la population ou encore à l'accroissement des incertitudes économiques et politiques au niveau mondial. Pendant près d'une décennie à travailler au Bureau chargé de la Finlande à l'OCDE, j'ai été impressionné par la rationalité et le pragmatisme dont, en Finlande, les responsables de l'action publique et les acteurs économiques au sens large font preuve lorsqu'ils s'efforcent de relever les défis, ainsi que par leur aptitude à forger un consensus sur des questions décisives. J'ai donc la conviction que la Finlande accomplira autant dans les cinquante années à venir que depuis 1969 et qu'elle continuera à inspirer les membres de l'OCDE et les autres dans leur quête de politiques meilleures pour une vie meilleure.

@L'Observateur de l'OCDE, janvier 2019

\section{Références}

OOCDE (2018), Études économiques de l'OCDE : Finlande 2018 (version abrégée), Éditions OCDE, Paris, http://dx.doi.org/10.1787/eco_surveys-fin-2018-en

Tableau de bord européen de l'innovation 2018 à l'adresse https://ec.europa.eu/ growth/industry/innovation/facts-figures/scoreboards_en 